\title{
UKRN Position on Bureaucracy in Research
}

The UK Reproducibility Network Steering Group $15^{\text {th }}$ June 2021

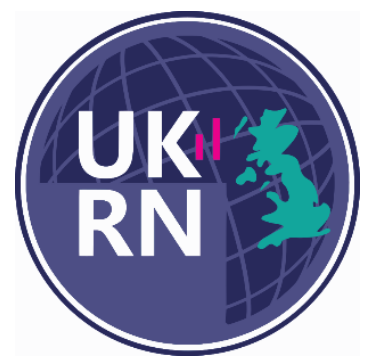

We welcome efforts to identify ways of reducing research bureaucracy and unnecessary burdens placed on researchers, supporting them to focus on research itself ${ }^{1}$. In particular, we are pleased to see reference to a "system-wide perspective".

However, we would also urge consideration of the impact of change across whole research lifecycle. Reducing apparent bureaucracy may actually reduce efficiency if this has a negative impact on the quality of research outputs at a later stage of the research process.

With this in mind, we encourage consideration of the following:

1. Strategic and central investment in infrastructure that promotes research quality, and transparency in research processes (e.g., data repositories, study registration platforms).

2. Creation of a flexible but secure career structure from PhD student to senior lecturer, to allow researchers to spend more time focused on research and less on securing their future employment (c.f. changes to medical career structures from the Calman Report ${ }^{2}$ forwards).

3. Development of "quality by design" systems (i.e., institutional systems for evaluating and improving research design, conduct analysis, and reporting), in place of audit for accountability (e.g., REF), as a means of ensuring research quality.

4. Consideration of the appropriate balance between funding from competitive grants versus support for research as part of core institutional funding, alongside mechanisms to improve the efficiency of grant review (e.g., wider use of preliminary applications).

5. Leveraging data analytics at scale to provide relevant institution-level measures of performance (e.g., Open Research performance using CWTS Leiden Ranking indicators; and the proportion of studies with a data availability statement, study registration, etc. as appropriate).

In our view, the goal should be for systems to be as light touch as possible whilst still being able to ensure value for money. These systems should support some framing of desirable characteristics of our research ecosystem (e.g., research culture, Equality Diversity and Inclusion, and increasing capacity in priority areas). This will require, in part, greater emphasis on ensuring robust processes and a positive research culture, and less emphasis on evaluating research outputs and individuals.

\section{${ }^{1}$ https://www.gov.uk/government/publications/review-of-research-bureaucracy/review-of- research-bureaucracy-terms-of-reference}

2 Department of Health (1993). Hospital doctors: training for the future. The report of the working group on specialist medical training. London: Department of Health.

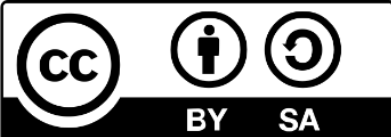

This work is licensed under a Creative Commons Attribution-ShareAlike 4.0 International License. 